\title{
Palliative sedation: Why we should be more concerned about the risks that patients experience an uncomfortable death
}

\author{
8 Q1 Reginald Deschepper ${ }^{\mathrm{a}, *}$, Steven Laureys ${ }^{\mathrm{b}}$, Said Hachimi Idrissi ${ }^{\mathrm{c}}$, Jan Poelaert ${ }^{\mathrm{d}}$, Johan Bilsen ${ }^{\mathrm{a}}$ \\ ${ }^{a}$ Department of Public Health, Vrije Universiteit Brussel, Brussel 1090, Belgium \\ ${ }^{\mathrm{b}}$ Coma Science Group, Cyclotron Research Centre and Neurology Department, University and University Hospital of Liège, Liège 4000, Belgium \\ ${ }^{c}$ Cerebral Resuscitation Research Group and the Centre of Neuroscience, Gent 9000, Belgium \\ ${ }^{\mathrm{d}}$ Department of Anesthesiology, Vrije Universiteit Brussel, Brussel 1090, Belgium
}

\section{Introduction}

Once death is imminent, a major concern of the family members and caregivers is to assure maximal comfort during this terminal phase. This can often be achieved by "conventional" pharmacological drugs such as opiates or other symptom-controlling drugs. However, in case of refractory symptoms leading to unbearable suffering such as intolerable pain, dyspnea, and delirium, a more drastic option may be chosen, known as palliative sedation (Table 1). In these cases, comfort is sought by reducing the patient's level of consciousness [12,23]. Although palliative sedation is ethically controversial and some studies have questioned its efficacy and safety [29], this practice has substantially increased. The incidence of palliative sedation is not easily measured, partly because there are several definitions and alternative terms in use, such as "terminal sedation" and "continuous sedation until death," to describe this practice [32]. However, the available studies indicate that the practice of palliative sedation is increasing in hospitals, nursing homes, and the home care setting. The overall reported incidences vary now between $7 \%$ and $17 \%$ of all deaths $[2,5]$. It is assumed that patients who are sedated according to the current standards of care and the guidelines of palliative sedation are unaware of their clinical situation and therefore do not experience symptoms of discomfort such as dyspnea, delirium, and other distressing conditions that are common during the terminal phase. However, a critical evaluation based on more recent evidence raises the question of whether the current assessments of suffering and awareness are accurate enough. Our concerns are based on 3 kinds of problems. Firstly, the assessment of comfort in dying patients is challenging; secondly, patients are sometimes mistakenly considered to be unaware; and thirdly, the titration of drugs is difficult.

\section{Problems with assessment of comfort in dying patients}

The gold standard for detecting distress is patient self-reporting. Several instruments, such as the Visual Analog Scale for Pain,

* Corresponding author. Address: Department of Public Health, Vrije Universiteit Brussel, Laarbeeklaan 103, Brussel 1090, Belgium. Tel.: +32 486632599 .

E-mail address: rdeschep@vub.ac.be (R. Deschepper). are based on this. However, in the case of palliative sedation, patients are usually unable to communicate whether or not they are still in distress or still (partially) aware of what is happening around them. Some scales have been developed for noncommunicative patients as well, such as the Critical Care Pain Observational Tool [18], the Behavioral Pain Scale [1], and the Richmond Agitation-Sedation Scale [3], but several problems have been reported. A well-documented problem is that these scales cannot detect pain and awareness in all patients; for example, because they depend on inferences made from patients' motor responsiveness [10,33]. Another problem is that these scales have been only partially validated for dying patients and, in most cases, not at all [4,8,31]. In the guidelines on palliative sedation, it is acknowledged that the efficacy and safety of palliative sedation is not sufficiently understood and that the usefulness of these observational scales has not been proven $[15,16]$. These findings cause even more concern considering the evidence that family members of patients, compared with caregivers, often have different perceptions of the patient's comfort and his/her quality of dying during palliative sedation. While family members tend to overestimate pain, caregivers often underestimate it [22]. Furthermore, assessment discrepancy between nurses and physicians often occurs $[6,17]$.

\section{Problems with (un)awareness}

In recent years, doubts have risen as to whether patients labeled "unconscious" really are completely insensate and unaware. Studies in different types of patients and settings that critically reviewed awareness have consistently reported that persons were, in contrast to what was assumed by the caregivers, not always (completely) unaware. For example, several studies showed that patients diagnosed as being in a vegetative state (now also called "unresponsive wakefulness syndrome") did show some (minimal) clinical signs of conscious awareness in about $40 \%$ of the cases [34]. In some cases, the purportedly unconscious patient could even reliably generate appropriate electroencephalographic responses to 2 distinct commands [14], and occasionally was even able to establish basic communication with "yes" or "no" answers using functional magnetic resonance imaging [28]. This proved that some minority of clinically diagnosed unresponsive patients had displayed at least some residual cognitive function and conscious 
Table 1

Core elements in guidelines on palliative sedation.

Indications for palliative sedation

- Refractory symptoms leading to unbearable suffering such as intolerable pain, dyspnea, and delirium $[11,12,23,35]$

Types of palliative sedation

- Degree: mild, intermediate, and deep [15]

- Continuity: from intermittent to continuous [15]

Ethical principles

- Palliative sedation is normal medical practice and must be clearly distinguished from the termination of life [35]

- Proportionality: the degree of sedation must not be deeper than necessary to relieve suffering $[11,12,23,35]$

- Palliative sedation will not (usually) hasten death (and that is certainly not the intention) $[12,15]$

Administration of drugs

- Titration to the minimum of level of consciousness reduction necessary to render symptoms tolerable $[16,23,24]$

- Lack of consensus

o "No good evidence exists to strongly recommend one medication over any other of those commonly used in continuous palliative sedation therapy" [16]

o "Midazolam is the drug of first choice" [24]

Monitoring of palliative sedated patients

- Aspects requiring monitoring: [16]

o Relief of suffering

o Level of consciousness (depth of sedation)

o Adverse side effects of sedation

- Guidelines' evaluations of the usefulness of monitoring scales

o "There are no scales available to assess the patient's comfort during continue sedation" [24]

o "Monitoring (observational) scales exist but the usefulness of these scales has not been proven" [16]

o "Presently no particular scale can be recommended" [16,24]

- "Scales involving administration of painful stimuli are not acceptable" [16]

- Frequency of monitoring: every 20 minutes until adequate sedation has been reached and then at least once a day [15,16]

- Nurses have the explicit task to observe, measure, and report [24]

awareness that even skilled caregivers were not able to recognize [25]. Also, patients with locked-in syndrome may be mistakenly considered unconscious, as may some (rare) patients during general anesthesia $[19,26]$. In contrast to the setting where surgical or intensive care patients are managed, advanced monitoring equipment is usually lacking in a palliative or home care setting. Palliative sedated patients ultimately die and therefore, patient self-reporting is also missing.

The above findings show that the "traditional" clinical tools and procedures to assess comfort and awareness in dying noncommunicative patients have important methodological limitations. It should be noted that the problems with assessments are not to be ascribed to lack of competence on the part of the caregivers, but are of a much more fundamental nature: the absence of reliable tools. The developers of guidelines are aware of these limitations and rightly point out that there is a lack of evidence (Table 1). Some guidelines mention that "there are no scales available to assess the patient's comfort" [24], and the authors of a recent guideline conclude that "presently no particular scale can be recommended" [16]. Sometimes guidelines refer to sedation scales, but point out that these scales are "not intended to measure the effect of sedation but to make clear when the sedation is too deep" [24]. The current guidelines for palliative sedation are therefore limited to suggesting "a daily visit by the physician" and "continue attention to possible expressions of discomfort (eg, facial expressions, movements, etc.)" [7,11,24]. Not surprisingly, nurses should also play an important role in signaling discomfort in sedated patients [24].

\section{Problems with the titration of drugs}

Since the aim of palliative sedation is to give optimal comfort but not to hasten death, the principle of proportionality is a pivotal aspect of this treatment and hence, the guidelines state that sedation should be "no deeper than necessary to avoid suffering" $[9,11,15,16,23,24]$. To meet this principle of proportionality, caregivers should carefully titrate the doses of the drugs so that they are high enough to provide comfort but should not hasten death. Studies have shown that palliative sedation does not usually affect survival time [27]. However, the fact that palliative sedation is considered by some to be "slow euthanasia" might lead physicians to be "extra careful" with the use of high doses of sedative medication [13]. Several studies have reported underuse of medicines due to a lack of knowledge, unwarranted beliefs, to avoid the perception of giving "excessive" doses, and even because of fear among caregivers of being accused of "killing" the patient $[21,30]$. In a Dutch study among nurses, the sedation was considered insufficiently effective by $42 \%$ of the respondents [7].

\section{How to improve assessments of suffering?}

Up until now, studies of the efficacy of palliative sedation to relieve pain and discomfort are based on observational scales or subjective assessments by caregivers $[7,29]$. Although some efforts have been made to validate the observation scales, as far as we know, all these attempts are based on the same paradigm, which is that all kinds of distress in all patients can be measured by observation of the patient, and that this is the only available method. However, in recent years, functional neuroimaging, such as functional magnetic resonance imaging, and encephalography have proven to be promising technologies for detecting awareness and pain that cannot be observed or detected by "traditional" methods $[20,28]$. Although these technologies also have their limitations and should not be regarded as a perfect surrogate for self-report, they provide valuable objective and quantifiable indicators of awareness and pain in noncommunicative patients $[20,28]$. Strikingly, they have not yet been used to check whether the current assessments of noncommunicative patients are reliable. It is remarkable that, given the increasing incidence of palliative sedation, there is so little concern about the risks that patients may experience an uncomfortable dying phase in which they are unable to signal their suffering. An assessment tool that would allow clinicians to more accurately determine the appropriate doses of medications would also encourage more vigorous symptom management in the dying.

Paradoxically, the inability to report distress might also be aggravated or even blocked by the use of drugs that might abolish potential further communication and even facial expressions [9]. 


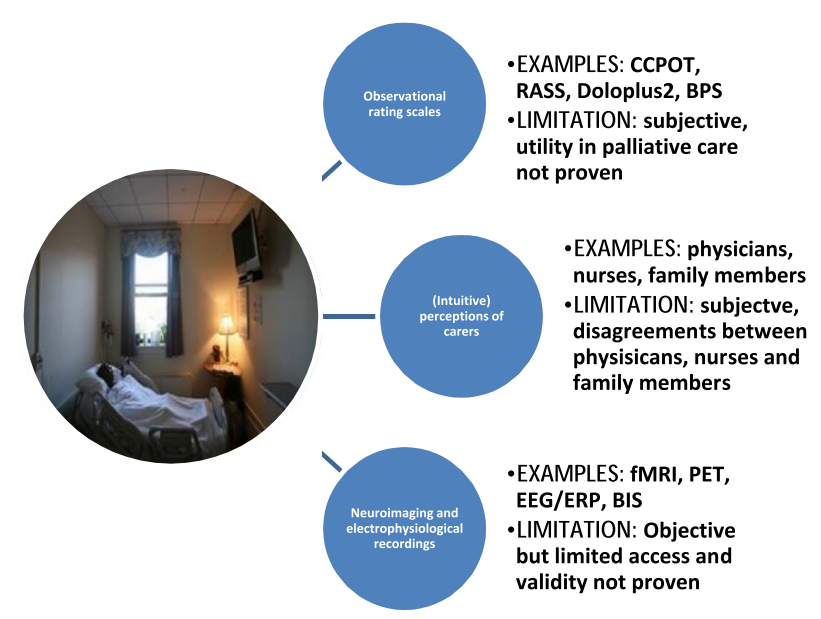

Fig. 1. Triangulation of assessment of distress in the noncommunicative dying patient. CCPOT, Critical Care Pain Observational Tool; RASS, Richmond AgitationSedation Scale; BPS, Behavioral Pain Scale; fMRI, functional magnetic resonance imaging; PET, positron emission tomography; ERP, event-related potential; EEG, electroencephalography; BIS, bispectral index.

Hence, some patients might have subjective phenomenological awareness or suffering with very limited, fluctuating or absent behavioral motor signs of distress [33]. The fact that neuroimaging or electrophysiology recordings have not been used so far to validate the assessment tools for distress in noncommunicative patients, even when doubts about these tools have arisen, may be related to the reluctance in palliative and end-of-life care to bother patients with high-tech equipment, as in most cases, patients have already experienced a long treatment period.

Dying uncommunicative patients are a vulnerable population and therefore, we should do everything possible to assure them a comfortable death free of pain and distress. We therefore urgently need a triangulation of methods in which existing observational scales, subjective assessments of caregivers and family, and neuroimaging and/or electrophysiological techniques are combined (Fig. 1). The latter are noninvasive procedures that should not burden too much the patient and his/her family. Due to the complexity and the intensity, this integrated mixed method is intended for research and not for everyday clinical assessments. It can be used for the validation of existing clinical tools for the assessment of distress in palliative sedated patients. Each of the 3 methods has its potentials and limitations, but combined they can be used to achieve the best possible assessments.

\section{Conflict of interest statement}

The authors have no conflicts of interest to disclose.

\section{Acknowledgements}

Supported by a grant from the Research Council of the Vrije Universiteit Brussel (Project HOA 27). S.L. is Research Director at the Belgian National Fund for Scientific Research (FRS).

\section{References}

[1] Ahlers SJ, van der Veen AM, van Dijk M, Tibboel D, Knibbe CA. The use of the Behavioral Pain Scale to assess pain in conscious sedated patients. Anesth Analg 2010;110:127-33.

[2] Anquinet L, Rietjens JA, Seale C, Seymour J, Deliens L, van der Heide A. The practice of continuous deep sedation until death in Flanders (Belgium), the Netherlands, and the UK: a comparative study. J Pain Symptom Manage 2012;44:33-43.
[3] Arevalo JJ, Brinkkemper T, van der Heide A, Rietjens JA, Ribbe M, Deliens L, Loer SA, Zuurmond WW, Perez RS. Palliative sedation: reliability and validity of sedation scales. J Pain Symptom Manage 2012;44:704-14.

[4] Arif-Rahu M, Grap MJ. Facial expression and pain in the critically ill noncommunicative patient: state of science review. Intensive Crit Care Nurs 2010;26:343-52.

[5] Bilsen J, Cohen J, Chambaere K, Pousset G, Onwuteaka-Philipsen BD, Mortier F, Deliens L. Medical end-of-life practices under the euthanasia law in Belgium. $\mathrm{N}$ Engl J Med 2009;361:1119-21.

[6] Breau LM, McGrath PJ, Stevens B, Beyene J, Camfield C, Finley GA, Franck L, Gibbins S, Howlett A, McKeever P, O'Brien K, Ohlsson A. Judgments of pain in the neonatal intensive care setting: a survey of direct care staffs' perceptions of pain in infants at risk for neurological impairment. Clin J Pain 2006;22:122-9.

[7] Brinkkemper T, Klinkenberg M, Deliens L, Eliel M, Rietjens JA, Zuurmond WW, Perez RS. Palliative sedation at home in the Netherlands: a nationwide survey among nurses. J Adv Nurs 2011;67:1719-28.

[8] Brinkkemper T, van Norel AM, Szadek KM, Loer SA, Zuurmond WW, Perez RS. The use of observational scales to monitor symptom control and depth of sedation in patients requiring palliative sedation: a systematic review. Palliat Med 2013;27:54-67.

[9] Broeckaert B, Mullie A, Gielen J, Desmet M, Declerck D, Vanden Berghe P Stuurgroep Ethiek FPZV. [Guideline palliative sedation sedation (Dutch)]. Available from: <http://www.pallialine.be/template.asp?f=rl_sedatie.htm>; 2012 [accessed xx.xx.xx].

[10] Brown JE, Chatterjee N, Younger J, Mackey S. Towards a physiology-based measure of pain: patterns of human brain activity distinguish painful from non-painful thermal stimulation. PLoS One 2011;6:e24124.

[11] Cherny NI, Radbruch L. European Association for Palliative Care (EAPC) recommended framework for the use of sedation in palliative care. Palliat Med 2009;23:581-93.

[12] Claessens P, Menten J, Schotsmans P, Broeckaert B. Palliative sedation: a review of the research literature. J Pain Symptom Manage 2008;36:310-33.

[13] Claessens P, Menten J, Schotsmans P. Broeckaert B. Level of consciousness in dying patients. The role of palliative sedation: a longitudinal prospective study. Am J Hosp Palliat Care 2012;29:195-200.

[14] Cruse D, Chennu S, Chatelle C, Bekinschtein TA, Fernandez-Espejo D, Pickard JD, Laureys S, Owen AM. Bedside detection of awareness in the vegetative state: a cohort study. Lancet 2011;378:2088-94.

[15] de Graeff A, Dean M. Palliative sedation therapy in the last weeks of life: a literature review and recommendations for standards. J Palliat Med 2007;10:67-85.

[16] Dean MM, Cellarius V, Henry B, Oneschuk D. Librach Canadian Society of Palliative Care Physicians Taskforce SL. Framework for continuous palliative sedation therapy in Canada. J Palliat Med 2012;15:870-9.

[17] Demertzi A, Schnakers C, Ledoux D, Chatelle C, Bruno MA, Vanhaudenhuyse A, Boly M, Moonen G, Laureys S. Different beliefs about pain perception in the vegetative and minimally conscious states: a European survey of medical and paramedical professionals. Prog Brain Res 2009;177:329-38.

[18] Gelinas C, Tousignant-Laflamme Y, Tanguay A, Bourgault P. Exploring the validity of the bispectral index, the critical-care pain observation tool and vital signs for the detection of pain in sedated and mechanically ventilated critically ill adults: a pilot study. Intensive Crit Care Nurs 2011;27:46-52.

[19] Ghoneim MM, Block RI, Haffarnan M, Mathews MJ. Awareness during anesthesia: risk factors, causes and sequelae: a review of reported cases in the literature. Anesth Analg 2009;108:527-35.

[20] Iannetti GD, Mouraux A. From the neuromatrix to the pain matrix (and back). Exp Brain Res 2010;205:1-12.

[21] Jackson DL, Proudfoot CW, Cann KF, Walsh TS. The incidence of sub-optimal sedation in the ICU: a systematic review. Crit Care 2009;13:R204.

[22] Kappesser J, Williams AC. Pain estimation: asking the right questions. PAIN ${ }^{\circledR}$ 2010;148:184-7.

[23] Kirk TW, Mahon MM. National Hospice and Palliative Care Organization (NHPCO) position statement and commentary on the use of palliative sedation in imminently dying terminally ill patients. J Pain Symptom Manage 2010;39:914-23.

[24] KNMG. Guideline for palliative sedation. Available at: <http:// knmg.artsennet.nl/Publicaties/KNMGpublicatie/Guideline-for-palliativesedation-2009.htm>; 2009. [accessed xx.xx.xx].

[25] Laureys S, Schiff ND. Coma and consciousness: paradigms (re)framed by neuroimaging. Neuroimage 2012;61:478-91.

[26] Leon-Carrion J, Van EP, Dominguez-Morales MR, Perez-Santamaria FJ. The locked-in syndrome: a syndrome looking for a therapy. Brain Inj 2002;16:571-82

[27] Maltoni M, Scarpi E, Rosati M, Derni S, Fabbri L, Martini F, Amadori D, Nanni O. Palliative sedation in end-of-life care and survival: a systematic review. J Clin Oncol 2012;30:1378-83.

[28] Monti MM, Vanhaudenhuyse A, Coleman MR, Boly M, Pickard JD, Tshibanda L, Owen AM, Laureys S. Willful modulation of brain activity in disorders of consciousness. N Engl J Med 2010;362:579-89.

[29] Morita T, Chinone Y, Ikenaga M, Miyoshi M, Nakaho T, Nishitateno K, Sakonji M, Shima Y, Suenaga K, Takigawa C, Kohara H, Tani K, Kawamura Y, Matsubara T, Watanabe A, Yagi Y, Sasaki T, Higuchi A, Kimura H, Abo H, Ozawa T, Kizawa Y, Uchitomi Y. Efficacy and safety of palliative sedation therapy: a multicenter, prospective, observational study conducted on specialized palliative care units in Japan. J Pain Symptom Manage 2005;30:320-8. 
[33] Sanders RD, Tononi G, Laureys S, Sleigh JW. Unresponsiveness not equal unconsciousness. Anesthesiology 2012;116:946-59.

[30] Oldenmenger WH, Sillevis Smitt PA, van DS, Stoter G, van der Rijt CC. A systematic review on barriers hindering adequate cancer pain management and interventions to reduce them: a critical appraisal. Eur J Cancer 2009;45:1370-80.

[31] Pudas-Tahka SM, Axelin A, Aantaa R, Lund V, Salantera S. Pain assessment tools for unconscious or sedated intensive care patients: a systematic review. J Adv Nurs 2009;65:946-56.

[32] Rys S, Mortier F, Deliens L, Deschepper R, Battin MP, Bilsen J. Continuous sedation until death: moral justifications of physicians and nurses-a content analysis of opinion pieces. Med Health Care Philos 2012 [Epub ahead of print].

[34] Schnakers C, Vanhaudenhuyse A, Giacino J, Ventura M, Boly M, Majerus S, Moonen G, Laureys S. Diagnostic accuracy of the vegetative and minimally conscious state: clinical consensus versus standardized neurobehaviora assessment. BMC Neurol 2009;9:35.

[35] Verkerk M, Van WE, Legemaate J, de GA. A national guideline for palliative sedation in the Netherlands. J Pain Symptom Manage 2007;34:666-70. 